effectiveness of the intervention prior to knowing the quantitative outcomes of the trial, focusing on case study schools.

Methods The process evaluation design was guided by the UK Medical Research Council framework for the evaluation of complex interventions. Mixed methods included semi-structured interviews with head/depute head teachers and educational psychologists, ethnographic notes from reflexive discussion sessions, focus groups with pupils and staff, pupil and parent questionnaires. The preliminary analysis was carried out on ten case study schools.

Results SEED was delivered largely as intended, the main exception being fewer reflexive discussion and action cycles than intended. Participants cited restrictions on time and resources and a lack of embeddedness in local authority structures as barriers to implementation.

There was evidence that SEED was valued for providing time and structure to reflect on SEW and foster a collective commitment to tackle and prevent problems, although actions often reinforced existing priorities rather than encouraged the adoption of innovative initiatives. There was limited evidence of an improvement in pupils' SEW but there was evidence that SEED improved relationships between staff. The school's pre-existing climate, strength of leadership and readiness for change appeared important in determining engagement with SEED.

Conclusion Based on this preliminary case study analysis we were not confident that enough schools implemented the intervention in sufficient depth to demonstrate an effect on pupils' Strengths and Difficulties Questionnaire scores (primary outcome). However, the RCT subsequently found a significant improvement in pupil SEW across the life of the trial, particularly for older pupils. Ongoing process evaluation analyses aim to explain this and revisit key questions of implementation and mechanisms for change. These analyses will be available by September 2018.

\section{OP36 INDIVIDUAL, FAMILY AND SCHOOL-BASED INTERVENTIONS TO PREVENT MULTIPLE RISK BEHAVIOURS IN YOUNG PEOPLE AGED 8-25 YEARS: A COCHRANE SYSTEMATIC REVIEW AND META-ANALYSIS}

${ }^{1}$ GJ MacArthur, 'DM Caldwell, ${ }^{2} \mathrm{~J}$ Redmore, 'S Watkins, ${ }^{1} \mathrm{R}$ Kipping, ${ }^{3} \mathrm{~J}$ White, ${ }^{4} \mathrm{C}$ Chittleborough, ${ }^{1} \mathrm{R}$ Langford, ${ }^{1} \mathrm{~V}$ Er* ${ }^{*}{ }^{5} \mathrm{R}$ Lingam, ${ }^{6} \mathrm{~K}$ Pasch, ${ }^{1} \mathrm{DJ}$ Gunnell, ${ }^{1} \mathrm{M}$ Hickman, ${ }^{1} \mathrm{R}$ Campbell. 'Department of Population Health Sciences, University of Bristol, Bristol, UK; ${ }^{2}$ Medical School, University of Aberdeen School of Medicine and Dentistry, Aberdeen, UK; ${ }^{3}$ Centre for Trials Research, Cardiff University, Cardiff, UK; ${ }^{4}$ School of Public Health, Robinson Research Institute University of Adelaide, Adelaide, Australia; ${ }^{5}$ Institute of Health and Society, Newcastle University, Newcastle upon Tyne, UK; ${ }^{6}$ Department of Kinesiology and Health Education, University of Texas, Austin, USA

\subsection{6/jech-2018-SSMabstracts.36}

Background We aimed to undertake a Cochrane systematic review to quantify the effect of multiple risk behaviour interventions on prevention of substance use, antisocial behaviour, sexual risk, vehicle risk, self-harm, gambling, physical inactivity and unhealthy diet among individuals aged 8-25 years as little is known about their effectiveness (CD009927).

Methods Eleven databases were searched to 14 November 2016. Randomised controlled trials were included that addressed two or more risk behaviours in individuals aged 0 18 years. Data were pooled in a random-effects meta-analysis in Revman 5.3. For each outcome, we included subgroups relating to study type (individual, family or school-level and universal or targeted in approach). The quality of evidence was assessed using the GRADE approach.

Results We identified 34680 titles, screened 27691 articles and included seventy studies. We found moderate quality evidence indicating that universal school-level interventions were beneficial in relation to tobacco use (odds ratio [OR] 0.77, $95 \%$ confidence interval [CI] $0.60-0.97, \mathrm{n}=15 \quad 354, \mathrm{I}^{2} 57 \%$ ), alcohol use (OR 0.72, 95\% CI 0.56 to $0.92, \mathrm{n}=8,751, \mathrm{I}^{2}$ $58 \%$ ), and physical activity (OR $1.32,95 \%$ CI 1.16 to 1.50 , $\mathrm{n}=6441, \mathrm{I}^{2} 0 \%$ ) compared to a comparator. Lower quality evidence indicated possible benefit for drug use (OR 0.74, $95 \%$ CI 0.55 to $1.00, \mathrm{n}=11058, \mathrm{I}^{2} 69 \%$ ) and antisocial behaviour (OR $0.81,95 \%$ CI 0.66 to $0.98, \mathrm{n}=20 \quad 756, \mathrm{I}^{2}$ $66 \%$ ), while findings were less certain for sexual risk behaviour (OR 0.80, 95\% CI 0.60 to $1.08, \mathrm{n}=13$ 351, $\mathrm{I}^{2} 80 \%$; low quality evidence) and unhealthy diet (OR 0.82, 95\% CI 0.64 to $1.06, \mathrm{n}=6441, \mathrm{I}^{2} 49 \%$, moderate quality evidence). Analyses indicated that family- and individual-level interventions probably have little or no effect on these outcomes, although fewer such studies were identified. The quality of studies was judged to be of moderate or low quality for most outcomes, primarily owing to concerns around selection, performance and detection bias, and heterogeneity between studies.

Conclusion Available evidence is strongest for universal schoollevel interventions that target multiple risk behaviours demonstrating that they may be effective in preventing certain risk behaviours. However, concerns around poor reporting and study quality highlight the need to strengthen the evidence base in this field.

This abstract is based on preliminary findings from a Cochrane review currently underway. Upon completion and approval, the final version is expected to be published in the Cochrane Database of Systematic Reviews.

\section{\begin{tabular}{|l|l}
\hline OP37 DO INTERVENTIONS INTEGRATING HEALTH AND \\
\hline
\end{tabular} ACADEMIC EDUCATION IN SCHOOLS PREVENT SUBSTANCE MISUSE AND VIOLENCE? SYSTEMATIC REVIEW AND INTERVENTION COMPONENTS ANALYSIS}

${ }^{1} \mathrm{GJ}$ Melendez-Torres* ${ }^{2}{ }^{2} \mathrm{~T}$ Tancred, ${ }^{2} \mathrm{CP}$ Bonell. ${ }^{1} D E C I P H e r$, Cardiff University, Cardiff, UK; ${ }^{2}$ Department of Social and Environmental Health Research, London School of Hygiene and Tropical Medicine, London, UK

\subsection{6/jech-2018-SSMabstracts.37}

Background Increasingly constrained school timetables mean that there is often little space for specifically timetabled health education lessons. Interventions that integrate health education into academic lessons may prove more appealing to schools, and may be a promising means of addressing outcomes such as violence and substance use while also promoting academic attainment. This evidence has not yet been systematically reviewed. We synthesised evidence for these interventions' effectiveness and analysed their components.

Methods We searched 19 bibliographic databases and 32 websites. References were extracted from the reference lists of included studies and authors were contacted.We included reports with no restrictions on language or date. References were screened on title/abstract and those passing this screening were then screened on full report. Data extraction and appraisal were informed by the Cochrane risk of bias tool. Outcome evaluations were synthesised by key stage (KS) using multilevel meta-analysis where possible; otherwise, evidence was narratively synthesised. Components were analysed 